\title{
Spatially-resolved SFR in nearby disk galaxies using IFS data
}

\section{Catalán-Torrecilla ${ }^{1}$, A. Gil de Paz ${ }^{1}$, A. Castillo-Morales ${ }^{1}$, J. Méndez-Abreu ${ }^{2}$, S. Pascual ${ }^{1}$, T. Ruiz-Lara ${ }^{3}$, A. de Lorenzo-Cáceres ${ }^{2}$ and L. Sánchez-Menguiano ${ }^{3}$}

\footnotetext{
${ }^{1}$ Departamento de Astrofísica y CC. de la Atmósfera, Universidad Complutense de Madrid, E-28040, Madrid, Spain, email: ccatalan@ucm.es

${ }^{2}$ School of Physics and Astronomy, University of St. Andrews, SUPA, North Haugh, KY16 9SS, St. Andrews, UK

${ }^{3}$ Departamento de Física Teórica y del Cosmos, Universidad de Granada, Campus de Fuentenueva, E-18071 Granada, Spain
}

\begin{abstract}
Exploring the spatial distribution of the star formation rate (SFR) in nearby galaxies is essential to understand their evolution through cosmic time. With this aim in mind, we use a representative sample that contains a variety of morphological types, the CALIFA Integral Field Spectroscopy (IFS) sample. Previous to this work, we have verified that our extinction-corrected $\mathrm{H} \alpha$ measurements successfully reproduce the values derived from other SFR tracers such as $\mathrm{H} \alpha_{o b s}+\mathrm{IR}$ or $\mathrm{UV}_{\text {obs }}+\mathrm{IR}$ (Catalán-Torrecilla et al. 2015).

Now, we go one step further applying 2-dimensional photometric decompositions (MéndezAbreu et al. (2008), Méndez-Abreu et al. (2014)) over these datacubes. This method allows us to obtain the amount of SFR in the central part (bulge or nuclear source), the bar and the disk, separately. First, we determine the light coming from each component as the ratio between the luminosity in every component (bulge, bar or disk) and the total luminosity of the galaxy. Then, for each galaxy we multiply the IFS datacubes by these previous factors to recover the luminosity in each component. Finally, we derive the spectrum associated to each galaxy component integrating the spatial information in the weighted datacube using an elliptical aperture covering the whole galaxy.

$2 \mathrm{D}$ photometric decomposition applied over 3D datacubes will give us a more detailed understanding of the role that disks play in more massive galaxies. Knowing if the disks in more massive SF galaxies have on average a lower or higher level of star formation activity and how these results are affected by the presence of nuclear bars are still open questions that we can now solve. We describe the behavior of these components in the SFR vs. stellar mass diagram. In particular, we highlight the role of the disks and their contribution to both the integrated SFR for the whole galaxy and the SFR in the disk at different stellar masses in the SFR vs. stellar mass diagram together with their relative position to the star forming Main Sequence.
\end{abstract}

Keywords. techniques: spectroscopic, galaxies: spiral, evolution, star formation

\section{References}

Catalán-Torrecilla, C., Gil de Paz, A., Castillo-Morales, A., et al. 2015, A\&GA, 584, A87

Méndez-Abreu, J., Aguerri, J. A. L., Corsini, E. M., \& Simonneau, E. 2008, A\& A, 478, 353

Méndez-Abreu, J., Debattista, V. P., Corsini, E. M., \& Aguerri, J. A. L. 2014, A\& A, 572, A25 